\title{
MANIFOLDS MODELLED ON $R^{\infty}$ OR BOUNDED WEAK-* TOPOLOGIES
}

\author{
BY \\ RICHARD E. HEISEY $\left({ }^{1}\right)$
}

\begin{abstract}
Let $R^{\infty}=\lim _{\rightarrow} R^{n}$, and let $B^{*}\left(b^{*}\right)$ denote the conjugate, $B^{*}$, of a separable, infinite-dimensional Banach space with its bounded weak-* topology. We investigate properties of paracompact, topological manifolds $M, N$ modelled on $F$, where $F$ is either $R^{\infty}$ or $B^{*}\left(b^{*}\right)$. Included among our results are that locally trivial bundles and microbundles over $M$ with fiber $F$ are trivial; there is an open embedding $M \longrightarrow M \times F$; and if $M$ and $N$ have the same homotopy type, then $M \times F$ and $N \times F$ are homeomorphic. Also, if $U$ is an open subset of $B^{*}\left(b^{*}\right)$, then $U \times B^{*}\left(b^{*}\right)$ is homeomorphic to $U$. Thus, two open subsets of $B^{*}\left(b^{*}\right)$ are homeomorphic if and only if they have the same homotopy type. Our theorems about $B^{*}\left(b^{*}\right)$-manifolds, $B^{*}\left(b^{*}\right)$ as above, immediately yield analogous theorems about $B(b)$-manifolds, where $B(b)$ is a separable, reflexive, infinite-dimensional Banach space with its bounded weak topology.
\end{abstract}

0 . Introduction. Let $R^{\infty}=\lim _{\rightarrow} R^{n}$, and let $B^{*}\left(b^{*}\right)$ denote the conjugate, $B^{*}$, of a separable, infinite-dimensional Banach space with its bounded weak-* topology. The bounded weak-* topology is the finest topology agreeing with the weak-* topology on bounded sets. (The weak-* topology on $B^{*}$ is the smallest topology on which all the linear functionals $\{\hat{x} \mid x \in B\}$ are continuous where $\hat{x}(\alpha)=\alpha(x)$.) We investigate properties of paracompact, topological manifolds, $M, N$, modelled on $F$, where $F$ is either $R^{\infty}$ or $B^{*}\left(b^{*}\right)$. Included among our results are that locally trivial bundles and microbundles over $M$ with fiber $F$ are trivial; there is an open embedding $M \rightarrow M \times F$; and if $M$ and $N$ have the same homotopy type, then $M \times F$ is homeomorphic to $N \times F$. We also show that if $U$ is open in $B^{*}\left(b^{*}\right)$, then $U \times B^{*}\left(b^{*}\right)$ is homeomorphic to $U$. Thus, two open subsets of $B^{*}\left(b^{*}\right)$ are homeomorphic if and only if they have the same homotopy type.

Any theorem about $B^{*}\left(b^{*}\right)$-manifolds, $B^{*}\left(b^{*}\right)$ as above, yields an analogous

Received by the editors May 10, 1973 and, in revised form, April 17, 1974.

AMS (MOS) subject classifications (1970). Primary 58B05; Secondary 46A99.

Key words and phrases. Bounded weak-* topology, manifold, Banach space, topological vector space, direct limit, Hilbert cube, microbundle, bundle, stable classification.

(1) Part of this research was supported by a NASA Fellowship (1970-1971) and a Rotary Foundation Fellowship (1971-1972). This research constitutes part of the author's Ph. D. thesis at Cornell University. 
theorem about $B(b)$-manifolds where $B(b)$ is a separable, reflexive, infinite-dimensional Banach space with its bounded weak topology. This is because, if $B$ is separable and reflexive, then $B^{*}$ is separable, and the natural isomorphism $B \rightarrow$ $\left(B^{*}\right)^{*}$ identifies the bounded weak topology on $B$ with the bounded weak-* topology on $\left(B^{*}\right)^{*}$.

Interest in bounded weak topologies has arisen recently in global analysis. In $[14, \S 3]$ Richard Palais, commenting on work of J. Dowling, R. Graff and K. Uhlenbeck, observed that many Banach manifolds of sections possess a natural atlas such that the transition maps, in addition to being smooth, preserve the bounded weak topology. It is hoped that study of topological manifolds modelled on bounded weak topologies will add some insight to these manifolds with "extra structure".

The author gratefully acknowledges the generous advice and encouragement of his thesis advisor, David W. Henderson.

I. Notation. Our "spaces" are Hausdorff and our "maps" continuous. We use $B$ to denote a separable, infinite-dimensional Banach space, $B^{*}$ its conjugate, $b^{*}$ the bounded weak-* topology, and $B^{*}\left(b^{*}\right)$ the space $B^{*}$ with its $b^{*}$ topology. (Note [6, Corollary 5, p. 428] that $B^{*}\left(b^{*}\right)$ is a TVS (real topological vector space). We also write LCTVS for a locally convex TVS.) We let $I=[0,1], Q$ the Hilbert cube, which we regard as the countably infinite product of $J=[-1,1], R$ the reals, $R^{\infty}=\lim _{\rightarrow}\left\{R^{n}, i_{n}\right\}$ and $Q^{\infty}=\lim _{\rightarrow}\left\{Q^{n}, j_{n}\right\}$, where $i_{n}\left(x_{1}, \cdots, x_{n}\right)=$ $\left(x_{1}, \cdots, x_{n}, 0\right), j_{n}\left(x_{1}, \cdots, x_{n}\right)=\left(x_{1}, \cdots, x_{n}, 0\right)$.

II. Statement of results. First, we state a result from [8] that we will need.

TheOREM II. 1 ([8, TheOREM II. 6]). $B^{*}\left(b^{*}\right)$ is homeomorphic to $Q^{\infty}$. In particular, $B^{*}\left(b^{*}\right)$ is homeomorphic to $l_{2}(b)$.

Thus, any (topological) $B^{*}\left(b^{*}\right)$-manifold may be regarded as an $l_{2}(b)$-manifold. Since the canonical isomorphism $l_{2} \rightarrow l_{2}^{*}$ identifies the bounded weak and bounded weak-* topologies, we identify $l_{2}(b)$ with $l_{2}^{*}\left(b^{*}\right)$ when convenient.

Definition. Let $E$ be a TVS, $M$ an $E$-manifold and $X$ a space. A map $f: X \rightarrow M$ is said to be (linearly) split at $x \in X$ if there is a neighborhood $V$ of $f(x)$ and a homeomorphism of pairs $\phi:(V, V \cap f(X)) \rightarrow\left(V_{1} \times V_{2}, V_{1} \times\{0\}\right)$ where $V_{i}$ is open in $E_{i}, i=1,2$, and $E_{1}$ and $E_{2}$ are closed linear subspaces of $E$ such that $E$ is (linearly) homeomorphic to $E_{1} \times E_{2}$. If $f$ is (linearly) split at each $x \in X$, we say $f$ is (linearly) split.

For the remainder of this section, let $F$ denote either $B^{*}\left(b^{*}\right)$ or $R^{\infty}$. Let $M$ and $N$ denote paracompact, connected $F$-manifolds. We establish the following results. 
THEOREM II. 2. (a) There is a closed embedding $f: M \rightarrow F$. If $V$ is any neighborhood of $0 \in F$, we may take $f(M) \subset V$. (b) If $F=R^{\infty}$ or if $F=B^{*}\left(b^{*}\right)$ where there is a closed linear split embedding $B \oplus B \rightarrow B$, then there is a closed linearly split embedding $f: M \rightarrow F$.

Since $F$ is not first countable (see [9, Proposition III. 1(e)] for the case $F=B^{*}\left(b^{*}\right)$ ), it is not metrizable. Thus, $F$-manifolds cannot be ANR (metric). However, we do have:

THEOREM II. 3. If $f: M \rightarrow N$ is a closed split embedding, then $f(M)$ is a neighborhood retract of $N$.

COROLLARY II. 4. $M$ is homeomorphic to a closed neighborhood retract of $F$.

THEOREM II. 5. Any microbundle and any locally trivial bundle with base $M$ and fiber $F$ are trivial.

Our proof of Theorem II. 5 is patterned after proofs of Chapman in [3]. Chapman's hypotheses include that the fiber be homeomorphic to its countably infinite cartesian product. This is not true of $B^{*}\left(b^{*}\right)$ or $R^{\infty}$. However, both $B^{*}\left(b^{*}\right)$ and $R^{\infty}$ are homeomorphic to their countably infinite direct limit (combine Theorem II. 1 and [8, Corollary III. 3]). This was used crucially in establishing [8, Corollary II. 5], one of the main tools used in the proof of Theorem II. 5.

THEOREM II. 6. If $f: M \rightarrow N$ is a closed split embedding, then there is an open embedding $g: M \times F \rightarrow N \times F$ such that $g(m, 0)=(f(m), 0)$, each $m \in M$.

Corollary II. 7. There is an open embedding $M \times F \rightarrow F$.

DEFinition. Two embeddings $f, g: X \rightarrow Y, X$ and $Y$ spaces, are ambient invertibly isotopic if there is a level preserving homeomorphism (called an invertible isotopy) $h: Y \times I \rightarrow Y \times I$ such that $h(x, 0)=(x, 0)$ and $h(f(x), 1)=$ $(g(x), 1)$, all $x \in X$.

THEOREM II. 8. Any two homotopic closed embeddings $f, g: M \rightarrow N \times$ $\{0\} \subset N \times F$ are ambient invertibly isotopic in $N \times F$. Further, given any neighborhood $V$ of $0 \in F$, the invertible isotopy may be taken to be the identity on $N \times(F \backslash V) \times I$.

THEOREM II. 9 (STABle Classification). If $f: M \rightarrow N$ is a homotopy equivalence, then there is a homeomorphism $h: M \times F \rightarrow N \times F$ such that $h$ is homotopic to $f \times$ id.

The proofs of Theorems II. 6, II. 8, and II. 9 are modelled after proofs of 
Henderson in [11]. Henderson's theorems, however, assume that the model is a metrizable LCTVS homeomorphic to its countably infinite cartesian product. As mentioned above, $B^{*}\left(b^{*}\right)$ and $R^{\infty}$ are not metrizable and are not homeomorphic to their countable cartesian product. Also, since $B^{*}\left(b^{*}\right)$ and $R^{\infty}$ are not first countable, they cannot be open cones. In [10, Lemma 1.1] Henderson showed that any metrizable LCTVS is an open cone, and he used this crucially in establishing his stable classification theorem. The concept that takes the place of "being an open cone", here, is the property of having a basis of radially bounded starlike sets. §IV develops the needed auxiliary results related to this property.

The following theorem follows from work of Hansen [7] and T. tom Dieck [4] (see $\S \mathrm{X})$.

THEOREM II. 10. $M$ has the homotopy type of a CW-complex.

THEOREM II. 11. If $U$ is an open subset of $B^{*}\left(b^{*}\right)$, then $U \times B^{*}\left(b^{*}\right) \cong U$.

Our proof of Theorem II. 11 relies heavily on the work of Anderson and Schori [2] and Anderson and Chapman [1].

The following is immediate from Theorems II. 9 and II. 11.

COROLlaRY II. 12. Two open subsets of $B^{*}\left(b^{*}\right)$ are homeomorphic iff they have the same homotopy type.

III. Some preliminaries.

DEFINITION. We call a space $X$ a countable direct limit of compact metric spaces (CDLCMS) if $X=\bigcup\left\{C_{i} \mid i=1,2, \cdots\right\}$, where each $C_{i}$ is a compact metric space, $C_{i} \subset C_{i+1}$, and $O \subset X$ is open in $X$ iff $O \cap C_{i}$ is open in $C_{i}$, each $i$.

Examples are $R^{\infty}, Q^{\infty}$ and, e.g. by Theorem II. $1, B^{*}\left(b^{*}\right)$.

Proposition III. 1. Let $X$ be a CDLCMS. Then every subspace of $X$ is regular, separable, Lindelöf, and paracompact.

Proof of III. 1. By Proposition 4.3 of [7], $X$ is regular and Lindelöf. By a theorem of Morita [5, p. 174], $X$ is also paracompact. Since subspaces of regular spaces are regular, every subspace of $X$ is regular. Let $O$ be open in $X=$ $\bigcup\left\{C_{i} \mid i=1,2, \cdots\right\}$. Each $O \cap C_{i}$ is Lindelöf and locally compact, so $O \cap C_{i}=$ $\bigcup\left\{K_{i j} \mid j=1,2, \cdots\right\}$, where each $K_{i j}$ is the closure of a relatively compact neighborhood in $O \cap C_{i}$. Thus $O$ is the countable union of compact sets and hence Lindelöf. Now let $A$ be any subset of $X$, and let $\left\{V_{\alpha}=U_{\alpha} \cap A\right\}$ be an open cover of $A, U_{\alpha}$ open in $X$. Since $U=\bigcup\left\{U_{\alpha}\right\}$ is Lindelöf, there is a countable subcover of $\left\{U_{\alpha}\right\}$, and hence also of $\left\{V_{\alpha}\right\}$. Thus, every subspace of $X$ is Lindelöf. By Morita's theorem, again, every subspace of $X$ is paracompact. Finally, given 
$A \subset X, A \cap C_{i}$ is separable since it is metric and Lindelöf. Thus, $A$, the countable union of separable spaces, is separable.

Proposition III. 2. Let $F$ be a TVS which is a CDLCMS. Then any paracompact, connected F-manifold, $M$, is a CDLCMS.

Proof OF III. 2. Let $O$ be open in $F, F=\bigcup\left\{C_{i} \mid i=1,2, \cdots\right\}$. We have $O \cap C_{i}=\bigcup\left\{K_{i j}\right\}$ as in the proof of Proposition III. 1. Since $O=\lim _{\rightarrow}\left(O \cap C_{i}\right)$, it follows that $O$ is a CDLCMS. By Proposition III. 1 each open subset of $F$ is Lindelöf. Thus, by [9, Proposition III. 4] $M$ is Lindelöf. Thus, $M=\bigcup\left\{O_{i} \mid i=\right.$ $1,2, \cdots\}$ where each $O_{i}$ is homeomorphic to an open subset of $F$. Since each $O_{i}$ is a CDLCMS, it follows easily that $M$ is also.

Proposition III. 3. Let $X$ be a CDLCMS, F a LCTVS. Then any map $f: A \rightarrow F, A$ closed in $X$, extends to a map $f^{\prime}: X \rightarrow F$ such that $f^{\prime}(X) \subset$ convex hull $(f(A))$.

Proof of III. 3. Using Dugundji's extension theorem [5, p. 188] in place of Tietze's extension theorem, the proof is like that of [7, Proposition 4.3(i)].

IV. Radially bounded starlike sets. If $F$ is a TVS and $y \in F \backslash\{0\}$ let $R_{y}=$ $\{\alpha y \mid \alpha \in[0, \infty)\}$, the ray through $y$. If $W \subset F$ and $\alpha \in R$, let $\alpha W=\{\alpha w \mid w \in W\}$.

Definition. A starlike set in a TVS $F$ is a neighborhood $U$ of the origin such that each ray from the origin intersects $U$ in a connected interval and intersects $\operatorname{Bd}(U)$, the topological boundary of $U$, in at most one point.

It is an easy exercise to show that any convex neighborhood of the origin is starlike.

DEFINITION. A subset of TVS is radially bounded if it intersects each ray (from the origin) in a bounded set.

Recall that a set $C$ in a TVS is circled if $\lambda C \subset C$, all $|\lambda|<1$.

THEOREM IV. 1. $B^{*}\left(b^{*}\right)$ and $R^{\infty}$ have bases at the origin of radially bound$e d$, circled, convex (and hence starlike) neighborhoods.

PROOF OF IV. 1. Since $R$ is locally compact, it follows that $\left\{\left[\Pi_{i=1}^{\infty}\left(-\alpha_{i}, \alpha_{i}\right)\right] \cap R^{\infty} \mid \alpha_{i}>0, \alpha_{i} \rightarrow 0\right\}$ is a basis at the origin in $R^{\infty}$; and each set in this collection is radially bounded, circled and convex.

By the Banach-Dieudonne theorem [6, Lemma 4, p. 427], sets of the form $\left\{x^{*} \in B^{*}|| x^{*}\left(x_{i}\right)\left|<\alpha_{i}, i=1,2, \cdots,\left\|x_{i}\right\|=1,\right| \alpha_{i} \mid \rightarrow \infty\right\}$ are a basis at the origin in $B^{*}\left(b^{*}\right)$. Since $B$ is separable metric, there is a sequence $\left\{d_{i} \mid i=1,2\right.$, $\cdots\} \subset\{x \in B \mid\|x\|=1\}$ such that the closure of $\left\{d_{i}\right\}$ is $\{x \in B \mid\|x\|=1\}$. Given 


$$
U=\left\{x^{*} \in B^{*}|| x^{*}\left(x_{i}\right)\left|<\alpha_{i}, i=1,2, \cdots,\left\|x_{i}\right\|=1,\right| \alpha_{i} \mid \rightarrow \infty\right\},
$$

let $V=\left\{x^{*} \in B^{*}|| x^{*}\left(y_{i}\right) \mid<\beta_{i}, i=1,2, \cdots\right\}$ where $y_{i}=x_{(i+1) / 2}, \beta_{i}=$ $\alpha_{(i+1) / 2}$ if $i$ is odd and $y_{i}=d_{i / 2}, \beta_{i}=i$ if $i$ is even. Then $V$ is a convex, circled neighborhood of $0 \in B^{*}\left(b^{*}\right)$ contained in $U$. Also, $V$ is radially bounded. To see this, let $x^{*} \in B^{*} \backslash\{0\}$. Then $\left|x^{*}\left(d_{j}\right)\right|=\delta>0$, some $j$. If $\alpha>(2 j) / \delta$, then $\left|\left(\alpha x^{*}\right)\left(y_{2 j}\right)\right|=\left|\left(\alpha x^{*}\right)\left(d_{j}\right)\right|>2 j$, so that $\alpha x^{*} \notin V$.

Notation. We write "RBS" for "radially bounded starlike".

Note that the boundary of a RBS set intersects each ray in exactly one point.

Definition. Let $V$ be a RBS set in the TVS $F$. Define $\mu_{V}: F \backslash\{0\} \rightarrow$ $F \backslash\{0\}$ by letting $\mu_{V}(x)$ be the unique point in $\operatorname{Bd}(V) \cap R_{x}$. Define $\gamma_{V}: F \backslash\{0\}$ $\rightarrow(0, \infty)$ by $x=\gamma_{V}(x) \mu_{V}(x)$.

Note $x \in V$ iff $\gamma_{V}(x)<1$, and $\gamma_{V}(\alpha x)=\alpha \gamma_{V}(x)$ all $\alpha>0$.

LEMMA IV. 2. If $V$ is a RBS set in the TVS F, then $\mu_{V}: F \backslash\{0\} \rightarrow F \backslash\{0\}$ and $\gamma_{V}: F \backslash\{0\} \rightarrow(0, \infty)$ are continuous.

PROOF OF IV. 2 (adapted from an unpublished argument of David Henderson). Let $\mu=\mu_{V}, \gamma=\gamma_{V}$. Since $\mu(x)=(1 / \gamma(x)) x$, we need only prove that $\gamma$ is continuous. Fix $x_{0} \in F \backslash\{0\}$, and let $0<\delta<\gamma\left(x_{0}\right)$. If $x_{0}$ were a limit point of $A=\left\{x \in F \backslash\{0\} \mid \gamma\left(x_{0}\right)-\gamma(x)>\delta\right\}$, then $\left(1 / \gamma\left(x_{0}\right)\right) x_{0}=\mu\left(x_{0}\right)$ would be a limit point of $\left(1 / \gamma\left(x_{0}\right)\right) A$. But $\left(1 / \gamma\left(x_{0}\right)\right) A \subset\left(1-\delta^{\prime}\right) V$, where $\delta^{\prime}=\delta / \gamma\left(x_{0}\right)<1$, so this is impossible. Thus, $x_{0}$ is not a limit point of $A$. Similarly, $x_{0}$ is not the limit point of $\left\{x \in F \backslash\{0\} \mid \gamma(x)-\gamma\left(x_{0}\right)>\delta\right\}$, and continuity of $\gamma$ follows.

Definition. Let $B$ be a space, $F$ a TVS. Let $W$ be an open subset of $B \times F$ such that for each $b \in B, W_{b}=W \cap(b \times F)$, regarded as lying in $F$, is a RBS set. For each $b \in B$, let $\mu_{b}=\mu_{W_{b}}: F \backslash\{0\} \rightarrow F \backslash\{0\}$ and $\gamma_{b}=\gamma_{w_{b}}$ : $F \backslash\{0\} \rightarrow(0, \infty)$ be as in Lemma IV. 2 . We call $W$ a radially bounded starlike tube (abbreviated RBS tube) if $(b, x) \rightarrow \mu_{b}(x)$ and $(b, x) \rightarrow \gamma_{b}(x)$ are continuous on $B \times(F \backslash\{0\})$. Each $W_{b}$ will be called a fiber of $W$.

By Lemma IV. 2 RBS sets may be regarded as special cases of RBS tubes.

Given $A \subset B \times F$ and $\alpha>0$, we let $\alpha A=\{(b, \alpha x) \mid(b, x) \in A\}$.

Proposition IV. 3. Let $W \subset B \times F$ be a RBS tube. Then there is a homeomorphism $h: B \times F \rightarrow W$ such that $h$ is the identity on $1 / 2 W, h$ is fiber preserving and on each fiber is ray preserving, and $h(B \times \bar{V})$ is closed in $B \times F$ for every $R B S$ set $V \subset F$.

Proof of IV. 3. Let $\delta:[0, \infty) \rightarrow[0,1)$ be any homeomorphism which is the identity on $[0,1 / 2]$. Define $h: B \times F \rightarrow W$ by $h(b, x)=\left(b, \delta\left(\gamma_{b}(x)\right) \mu_{b}(x)\right)$, 
$x \neq 0$, and $h(b, 0)=(b, 0)$. Note that $h^{-1}(b, x)=\left(b, \delta^{-1}\left(\gamma_{b}(x)\right) \mu_{b}(x)\right), x \neq 0$. Since $W$ is a RBS tube, both $h$ and $h^{-1}$ are continuous. Clearly, $h$ is the identity on $1 / 2 W$, is fiber preserving and is ray preserving on each fiber.

Let $V \subset F$ be a RBS set, and let $(b, x) \in(B \times F) \vee(B \times \bar{V})$. Then $(b, s x)$ $\in W \backslash h(B \times \bar{V})$, some $s \leqslant 1$, so there are neighborhoods $O$ of $b$ in $B$ and $U$ of $s x$ in $F$ such that $0 \times U \subset W \backslash h(B \times \bar{V})$. Then $(b, x) \in 0 \times(1 / s) U \subset$ $(B \times F) \backslash h(B \times \bar{V})$.

Proposition IV. 4. Let $B$ be a paracompact space, $F$ a TVS with a basis at the origin consisting of RBS sets. Then any neighborhood $U$ of $B \times\{0\}$ in $B \times F$ contains a RBS tube.

Proof OF IV. 4. Let $\left\{V_{\alpha}\right\}$ be a locally-finite cover of $B$ by open sets such that for each $\alpha$ there is a RBS set $W_{\alpha}$ in $F$ with $V_{\alpha} \times W_{\alpha} \subset U$. Let $\left\{\lambda_{\alpha}: B \rightarrow\right.$ $[0,1]\}$ be maps such that $\lambda_{\alpha}^{-1}((0,1]) \subset V_{\alpha}$ and finterior $\left.\lambda_{\alpha}^{-1}(1)\right\} \operatorname{cover} B$. Let

$$
W=\left\{(b, x) \in B \times F \mid x \in \bigcup\left\{\lambda_{\alpha}(b) W_{\alpha}\right\}\right. \text {. }
$$

Then $W$ is open and each $W_{b}$ is the finite union of RBS sets and hence is a RBS set. For each $b \in B$, let $\mu_{b}: F \backslash\{0\} \rightarrow F \backslash\{0\}$ and $\gamma_{b}: F \backslash\{0\} \rightarrow(0, \infty)$ be the maps associated with $W_{b}$, and for each $\alpha$ let $\mu_{\alpha}$ and $\gamma_{\alpha}$ be the maps associated with $W_{\alpha}$. Fix $(a, y) \in B \times(F \backslash\{0\})$. Let $G$ be a neighborhood of a meeting only, say, $V_{\alpha_{1}}, \cdots, V_{\alpha_{n}}$. Then, if $(b, x) \in G \times(F \backslash\{0\}), \mu_{b}(x)=\max \left\{\lambda_{\alpha_{i}}(b) \mu_{\alpha_{i}}(x) \mid\right.$ $i=1, \cdots, n\}$, so that $(b, x) \rightarrow \mu_{b}(x)$ is continuous. The continuity of $(b, x)$ $\rightarrow \gamma_{b}(x)$ now follows since $1 / \gamma_{b}(x)=\gamma_{a}\left(\mu_{b}(x)\right) / \gamma_{a}(x)$.

THEOREM IV. 5. Let $B$ be a space, $F$ a TVS. Let $W_{1}, W_{2}, W_{3}, W_{4}$ be RBS tubes in $B \times F$ with $\bar{W}_{1} \subset W_{2} \subset W_{3} \subset \bar{W}_{3} \subset W_{4}$. Then there is a homeomorphism $s=s\left(W_{1}, W_{2}, W_{3}, W_{4}\right): B \times F \rightarrow B \times F$ carrying $W_{2}$ onto $W_{3}$ such that $s$ is fiber preserving, ray preserving on each fiber, and the identity on $\bar{W}_{1} \cup\left[(B \times F) \backslash W_{4}\right]$. Further, $s$ is ambient invertibly isotopic to the identity by an invertible isotopy which is the identity on $\left(\bar{W}_{1} \cup\left[(B \times F) \backslash W_{4}\right]\right) \times I$.

Proof of IV. 5. First assume the $W_{i}$ are RBS sets. For $i=1,2,3,4$ let $\mu_{i}: F \backslash\{0\} \rightarrow F$ and $\gamma_{i}: F \backslash\{0\} \rightarrow(0, \infty)$ be the maps associated with $w_{i}$. Regard the elements on each ray of $F$ as ordered via a homeomorphism onto $[0, \infty)$. Then $\mu_{1}(x)<\mu_{2}(x) \leqslant \mu_{3}(x)<\mu_{4}(x)$ and $\gamma_{1}(x)>\gamma_{2}(x) \geqslant \gamma_{3}(x)>\gamma_{4}(x)$. For each $x \in F \backslash\{0\}$ let

$$
\begin{array}{ll}
a(x)=\gamma_{4}(x) / \gamma_{1}(x), & b(x)=\gamma_{4}(x) / \gamma_{2}(x), \\
c(x)=\gamma_{4}(x) / \gamma_{3}(x), & d(x)=\gamma_{4}(x) / \gamma_{4}(x)=1 .
\end{array}
$$


On each ray the maps $a, b, c$, and $d$ are constant, since $\gamma_{i}(r x)=r \gamma_{i}(x)$, and $a<$ $b \leqslant c<d$. Also, on $\operatorname{Bd}\left(W_{1}\right), \gamma_{1}(x)=1$ so that $a(x)=\gamma_{4}(x)$, etc. For $t \in I$ define $h_{t}: F \rightarrow F$ by

$$
h_{t}=\left\{\begin{array}{l}
\text { identity on } \bar{W}_{1} \cup\left(F \backslash W_{4}\right), \\
{\left[a+\frac{\gamma_{4}-a}{b-a}([b+t(c-b)]-a)\right] \mu_{4} \text { on } \bar{W}_{2} \backslash W_{1},} \\
{\left[b+t(c-b)+\frac{\gamma_{4}-b}{1-b}(1-[b+t(c-b)])\right] \mu_{4} \text { on } \bar{W}_{4} \backslash W_{2} .}
\end{array}\right.
$$

Then $h_{t}$ is a homeomorphism with inverse

$$
h_{t}^{-1}=\left\{\begin{array}{l}
\text { identity on } \bar{W}_{1} \cup\left(F \backslash W_{4}\right), \\
{\left[a+\frac{\gamma_{4}-a}{b+t(c-b)-a}(b-a)\right] \mu_{4} \text { on } h_{t}\left(\bar{W}_{2}\right) \backslash W_{1},} \\
{\left[b+\frac{\gamma_{4}-[b+t(c-b)]}{1-[b+t(c-b)]}(1-b)\right] \mu_{4} \text { on } \bar{W}_{4} \bigvee_{t}\left(W_{2}\right) .}
\end{array}\right.
$$

Define $h: F \times I \rightarrow F \times I$ by $h(x, t)=\left(h_{t}(x), t\right)$. Then $h_{1}$ is the required homeomorphism $s\left(W_{1}, W_{2}, W_{3}, W_{4}\right)$, and $h$ is the required invertible isotopy.

Now, given RBS tubes $W_{1}, W_{2}, W_{3}, W_{4}$, the required isotopy is $S: B \times F \times I \rightarrow$ $B \times F \times I$ defined by $S(b, x, t)=\left(b,\left(h_{b}\right)_{t}(x), t\right)$ where, for each $b \in B,\left(h_{b}\right)_{t}$ is the homeomorphism constructed above for the RBS sets $\left(W_{1}\right)_{b}, \cdots,\left(W_{4}\right)_{b}$.

Definition. We say that two embeddings $f, g: X \rightarrow Y$ are isotopic if there is a homotopy, called an isotopy, $H: X \times I \rightarrow Y$ such that $H_{0}=f, H_{1}=g$, and $H_{t}$ is an embedding, $t \in I$.

The following lemma and its proof, using our Theorem IV. 5, are direct analogues of Lemma 5.1 in [11].

LEMMA IV. 6. Let $B$ be a paracompact space, $F$ a TVS having $a$ basis at the origin of RBS sets. Let $A \subset F$ be a fixed RBS set. For each positive integer $i$, let $f_{i}: B \times F \rightarrow B \times F$ be an open embedding such that $f_{i}$ is the identity on $B \times\{0\}, f_{i}(B \times F) \subset B \times A$, and $f_{i}(B \times \bar{V})$ is closed in $B \times F$ for each $R B S$ set $V \subset F$. Then there is a homeomorphism of $B \times F$ onto the direct limit of

$$
B \times F \stackrel{f_{1}}{\longrightarrow} B \times F \stackrel{f_{2}}{\longrightarrow} B \times F \stackrel{f_{3}}{\longrightarrow} \cdots
$$

which is isotopic to the inclusion of the first $B \times F$ into the direct limit.

V. Proof of Theorem II. 2.

LEMma V. 1. Let $F$ be $R^{\infty}$ or $B^{*}\left(b^{*}\right)$, and let $V$ be any neighborhood of $0 \in F$. Then there is a closed embedding $f: F \rightarrow F$ with $f(F) \subset V$. 
Proof of V. 1. If $F=B^{*}\left(b^{*}\right)$, by Theorem II. 1 there is a homeomorphism $\alpha: F \rightarrow Q^{\infty}$. We may assume $\alpha(0)=(0,0, \cdots)$. By [8, Proposition II. 1(a)], $\alpha(V) \supset\left(U_{1} \times U_{2} \times \cdots\right) \cap Q^{\infty}$ for some sequence $\left\{U_{i}\right\}$ of neighborhoods of $0 \in Q$. Let $h_{i}: Q \rightarrow U_{i}$ be a (closed) embedding such that $h_{i}(0)=0$. Then $h=$ $\left(h_{1}, h_{2}, \cdots\right): Q^{\infty} \rightarrow Q^{\infty}$ is a closed embedding with $h\left(Q^{\infty}\right) \subset \alpha(V)$. The required embedding is $f=\alpha^{-1} f \alpha$. If $F=R^{\infty}$, observe that $R^{\infty}=\lim _{\vec{Q}} B_{n}^{n}$, where $B_{n}^{n}=\{x \in R \mid\|x\| \leqslant n\}$. Hence, an argument like that given for $\vec{Q}^{\infty}$ establishes the existence of $f$.

We regard $l_{2}$ as all real sequences $x=\left\{x_{i}\right\}$ such that $\|x\|^{2}=\Sigma\left|x_{i}\right|^{2}<\infty$.

Lemma V. 2. The natural bijections $R^{\infty} \times R^{\infty} \rightarrow R^{\infty}$ and $l_{2}(b) \times l_{2}(b) \rightarrow$ $l_{2}(b)$ given by $\left(\left(x_{1}, x_{2}, \cdots\right),\left(y_{1}, y_{2}, \cdots\right)\right) \rightarrow\left(x_{1}, y_{1}, x_{2}, y_{2}, \cdots\right)$ are homeomorphisms.

Proof OF V. 2. Since $R$ is locally compact, sets of the form $\left[\Pi_{1}^{\infty}\left(-\alpha_{i}, \alpha_{i}\right)\right]$ $\cap R^{\infty}, \alpha_{i}>0, \alpha_{i} \rightarrow 0$, form a basis at the origin in $R^{\infty}$. By the Banach-Dieudonné theorem [6, Lemma 4, p. 427] a basis at the origin for $l_{2}(b)$ is all sets of the form

$$
\left\{x \in l_{2}||\left(x, y_{i}\right) \mid<1, y_{i} \in l_{2},\left\|y_{i}\right\| \rightarrow 0, i=1,2, \cdots\right\} .
$$

The lemma follows immediately.

Proof of Theorem II. 2. Assume first that $F=B^{*}\left(b^{*}\right)$ where there is a closed linear split embedding $B \oplus B \rightarrow B$. In the notation of [9], any $F$-manifold is a $\left(C^{-1}, b^{*}\right)$-manifold modelled on $B^{*}$. By [9, Theorem II. 4] there is a closed split $\left(C^{-1}, b^{*}\right)$-embedding $f: M \rightarrow B^{*}$, and this $f$ is a closed linearly split (topological) embedding $M \rightarrow F$. This establishes (b). For (a) regard $M$ as an $l_{2}(b)$ manifold by Theorem II. 1, apply (b) and then Lemma V. 1.

Now assume $F=R^{\infty}$. By Lemma V. 2 there is a linear homeomorphism $R^{\infty} \times R^{\infty} \rightarrow R^{\infty}$, and by Propositions III. 1 and III. $2 M$ is regular and Lindelöf. A proof like the proof of Theorem II. 3 in [9] now gives a closed, linearly split embedding $f: M \rightarrow R^{\infty}$. This proves (b), and (a) follows by applying Lemma V. 1 .

\section{Proofs of Theorem II. 3 and Corollary II. 4.}

Lemma VI. 1. Let $M, N, f: M \rightarrow N$ be as in Theorem II. 3. Then there is a locally-finite collection $\left\{U_{i} \mid i=1,2, \cdots\right\}$ of open subsets of $N$ covering $f(M)$ such that each $\left(U_{i}, U_{i} \cap f(M)\right)$ is pairwise homeomorphic to $\left(O_{i} \times F_{i}, O_{i} \times 0\right)$, where $O_{i}$ is open in $E_{i}$ and $E_{i}$ and $F_{i}$ are closed linear subspaces of $F$ such that $F \cong E_{i} \times F_{i}$ (F as in $\left.\S \mathrm{II}\right)$.

Proof of VI. 1. By Theorem II. $1, F$ is a CDLCMS. By Propositions III. 1 and III. 2, every subspace of $N$ is Lindelöf and paracompact. Let $\left\{W_{i} \mid i=1,2\right.$, $\cdots$ b be a countable cover of $f(M)$ by open subsets of $N$ such that for each $i$ 
there is a homeomorphism $\phi_{i}:\left(W_{i}, W_{i} \cap f(M)\right) \rightarrow\left(D_{i} \times G_{i}, D_{i} \times 0\right)$, where $D_{i}$ is open in $E_{i}, G_{i}$ is open in $F_{i}$, and $E_{i}$ and $F_{i}$ are closed linear subspaces of $F$ such that $F \cong E_{i} \times F_{i}$. Let $\left\{V_{i}\right\}$ be a precise locally-finite refinement of $\left\{W_{i}\right\}$ in paracompact $\bigcup\left\{W_{i}\right\}$. Regard $\phi_{i}\left(V_{i} \cap f(M)\right)$ as a subspace of $E_{i}$, and note that it is paracompact. By Theorem IV. $1 F$, and hence $F_{i}$, has a basis at the origin of RBS sets. We have

$$
\begin{gathered}
\phi_{i}\left(V_{i} \cap f(M)\right) \times 0 \subset\left[\phi_{i}\left(V_{i} \cap f(M)\right) \times F_{i}\right] \cap \phi_{i}\left(V_{i}\right) \\
\subset \phi_{i}\left(V_{i} \cap f(M)\right) \times F_{i} .
\end{gathered}
$$

By Propositions IV. 3 and IV. 4 there is a RBS tube $S_{i}$ in $\left[\phi_{i}\left(V_{i} \cap f(M)\right) \times F_{i}\right] \cap$ $\phi_{i}\left(V_{i}\right)$ and a homeomorphism $\lambda_{i}: S_{i} \rightarrow \phi_{i}\left(V_{i} \cap f(M)\right) \times F_{i}$ which is the identity on $\phi_{i}\left(V_{i} \cap f(M)\right) \times 0$. Letting $U_{i}=\phi_{i}^{-1}\left(S_{i}\right)$ we obtain the required cover of $N$.

Proof of Theorem II. 3. Let $f: M \rightarrow N$ be a closed split embedding. Let $\left\{U_{i} \mid i=1,2, \cdots\right\}, E_{i}, F_{i}, O_{i}$ be as in Lemma VI. 1 , and let

$$
\phi_{i}:\left(U_{i}, U_{i} \cap f(M)\right) \rightarrow\left(O_{i} \times F_{i}, O_{i} \times 0\right)
$$

be a homeomorphism of pairs. Let $V_{i}=U_{i} \cap f(M)$.

Since $V_{1} \cup V_{2}$ is normal (Propositions III. 1 and III. 2) we can find $A_{1} \subset$ $V_{1}, A_{2} \subset V_{2}$ such that $A_{1}$ and $A_{2}$ are closed in $V_{1} \cup V_{2}$ (and hence in $U_{1} \cup$ $U_{2}$ ), and $V_{1} \cup V_{2}=\operatorname{Int} A_{1} \cup \operatorname{Int} A_{2}$, where Int $A_{i}$ is the interior of $A_{i}$ in $V_{1} \cup V_{2}, i=1$, 2. Again by normality, choose $G_{1}$ open in $U_{1} \cup U_{2}$ such that $A_{2} \subset G_{1} \subset \bar{G}_{1} \subset U_{2}$, where $\bar{G}_{1}$ is the closure of $G_{1}$ in $U_{1} \cup U_{2}$.

In what follows we identify subsets of $\mathrm{O}_{2} \times\{0\}$ with subsets of $\mathrm{O}_{2}$. Choose $G_{2}$ open in $\phi_{2}\left(A_{1} \cap A_{2}\right) \times F_{2}$ such that

$$
\phi_{2}\left(A_{1} \cap A_{2}\right) \subset G_{2} \subset \bar{G}_{2} \subset \phi_{2}\left(U_{1} \cap U_{2} \cap G_{1}\right) \cap\left[\phi_{2}\left(A_{1} \cap A_{2}\right) \times F_{2}\right],
$$

where $\bar{G}_{2}$ is the closure in $\phi_{2}\left(A_{1} \cap A_{2}\right) \times F_{2}$. Let $G_{3}$ be an open subset of $\phi_{2}\left(A_{2}\right) \times F_{2}$ such that $G_{2}=G_{3} \cap\left[\phi_{2}\left(A_{1} \cap A_{2}\right) \times F_{2}\right]$, and let $G_{4}=G_{3} \cap$ $\phi_{2}\left(A_{2}\right)$. Choose $G_{5}$ open in $\phi_{2}\left(A_{2}\right)$ such that $\phi_{2}\left(A_{1} \cap A_{2}\right) \subset G_{5} \subset \bar{G}_{5} \subset G_{4}$, where $\bar{G}_{5}$ is the closure in $\phi_{2}\left(A_{2}\right)$. Let

$$
G_{6}=\left(G_{3} \cup\left[\left(\phi_{2}\left(A_{2}\right) \backslash \bar{G}_{5}\right) \times F_{2}\right]\right) \cap \phi_{2}\left(G_{1}\right) .
$$

Then $G_{6}$ is a neighborhood of $\phi_{2}\left(A_{2}\right)$ in $\phi_{2}\left(A_{2}\right) \times F_{2}$. By Propositions IV. 3 and IV. 4 there is an open subset $G_{7}$ of $G_{6}$ such that $\phi_{2}\left(A_{2}\right) \subset G_{7} \subset \bar{G}_{7} \subset G_{6}$, where $\bar{G}_{7}$ is the closure in $\phi_{2}\left(A_{2}\right) \times F_{2}$, and a fiber preserving homeomorphism $\psi:\left(G_{7}, \bar{G}_{7}\right) \rightarrow\left(\phi_{2}\left(A_{2}\right) \times V, \phi_{2}\left(A_{2}\right) \times \bar{V}\right)$, where $\psi$ is the identity on $\phi_{2}\left(A_{2}\right) \times$ $0, V$ a RBS set in $F_{2}$, and $\bar{V}$ the closure of $V$ in $F_{2}$. Note that $\phi_{2}^{-1}\left(\bar{G}_{7}\right)$ is closed in $\bar{G}_{1}$, and hence also in $U_{1} \cup U_{2}$. 
Let $W_{2}=\left[U_{1} \backslash \phi_{2}^{-1}\left(\bar{G}_{7}\right)\right] \cup \phi_{2}^{-1}\left(G_{7}\right)$. Then $W_{2}$ is open in $U_{1} \cup U_{2}$ and contains $V_{1} \cup V_{2}$. We construct a retraction $r_{2}: W_{2} \rightarrow W_{2} \cap f(M)=V_{1} \cup V_{2}$. Let $\lambda: A_{2} \rightarrow[0,1]$ be a map such that $\lambda\left(A_{2} \backslash\right.$ Int $\left.A_{2}\right)=1$ and $\lambda\left(A_{2} \backslash A_{1}\right)=0$. Let Int $\phi_{2}\left(A_{2}\right)$ be the interior of $\phi_{2}\left(A_{2}\right)$ in $O_{2}$. Define $r_{2}^{\prime}: W_{2} \rightarrow U_{1} \cup U_{2}$ by

$$
r_{2}^{\prime}(x)= \begin{cases}\phi_{2}^{-1} \psi^{-1}\left(\phi_{2}(a), \lambda(a) v\right), & \text { if } x=\phi_{2}^{-1} \psi^{-1}(\phi(a), v) \in \phi_{2}^{-1} \psi^{-1}\left(\operatorname{Int} \phi_{2}\left(A_{2}\right) \times V\right), \\ x, & \text { otherwise. }\end{cases}
$$

Then $r_{2}^{\prime}$ is continuous, $r_{2}^{\prime}$ is the identity on $V_{1} \cup V_{2}$, and $r_{2}^{\prime}\left(W_{2}\right) \subset\left(U_{1} \cap W_{2}\right)$ $\cup V_{2}$. Let $I=r_{2}^{\prime}\left(W_{2}\right)$. Let $r_{1}: U_{1} \rightarrow V_{1}=U_{1} \cap f(M)$ be the retraction obtained by projection via the homeomorphism $\phi_{1}$. Define $r_{1}^{\prime}: I \rightarrow V_{1} \cup V_{2}$ by

$$
r_{1}^{\prime}(x)= \begin{cases}r_{1}(x), & \text { if } x \in I \cap U_{1}, \\ x, & \text { if } x \in \Lambda U_{1} .\end{cases}
$$

Then $r_{2}=r_{1}^{\prime} \circ r_{2}^{\prime}: W_{2} \rightarrow V_{1} \cup V_{2}$ is a retraction.

Inductively, as above, construct open subsets $W_{k}$ of $N$ such that $W_{k} \subset W_{k-1}$ $\cup U_{k}, W_{k} \supset V_{1} \cup \cdots \cup V_{k}, W_{k} \supset W_{k-1} \backslash U_{k}$ and then retractions $r_{k}: W_{k} \rightarrow$ $V_{1} \cup \cdots \cup V_{k}$ such that $r_{k}=r_{k-1}$ on $W_{k-1} \backslash U_{k}, k \geqslant 2$. Define $W$ and $r$ : $W \rightarrow f(M)$ as follows. Given $x \in \bigcup\left\{U_{i} \mid i=1,2, \cdots\right\}$, let $n(x)=\max \left\{i \mid x \in U_{i}\right\}$. Then $x \in W$ iff $x \in W_{n(x)}$. If $x \in W_{n(x)}$, then define $r(x)=r_{n(x)}(x)$. Then $W$ is a neighborhood of $f(M)$ in $N$, and $r: W \rightarrow f(M)$ is a retraction.

Proof of Corollary II. 4. If $F=B^{*}\left(b^{*}\right)$, we may regard $M$ as an $l_{2}^{*}\left(b^{*}\right)$-manifold. Thus, the corollary follows from Theorems II. 2(b) and II. 3.

\section{Proofs of Theorems II. 5 and II. 6 and Corollary II. 7.}

LEMMA VII. 1. Let $X$ be a paracompact space, $F$ a TVS having a basis at the origin of RBS sets (see §IV). If $A$ is closed in $X$ and $U$ is a neighborhood of $A \times 0$ in $A \times F$, then there is an X-preserving (i.e. $f(\{x\} \times F) \subset\{x\} \times F, x \in X)$ open embedding $f: X \times F \rightarrow X \times F$ such that $f(x, 0)=(x, 0)$ for each $x \in X$ and $f(A \times F) \subset U$.

Proof of VII. 1. Let $V$ be an open subset of $X \times F$ such that $V \cap(A \times$ $F)=U$. Let $W=V \cup((X \backslash A) \times F)$. By Proposition IV. $4 W$ contains a RBS tube. The desired embedding $X \times F \rightarrow X \times F$ is guaranteed by Proposition IV. 3 .

Definition (e.g. [5, p. 248]). A space is a $k$-space if it has the weak topology determined by the family of its compact subspaces.

If $Y=\left(Y, y_{0}\right)$ is a pointed space we denote by $Y^{\infty}$ the direct limit of the sequence 


$$
Y^{1} \stackrel{j_{1}}{\longrightarrow} Y^{2} \stackrel{j_{2}}{\longrightarrow} Y^{3} \stackrel{j_{3}}{\longrightarrow} \cdots
$$

where $j_{n}: Y^{n} \rightarrow Y^{n+1}$ is defined by $j_{n}\left(y_{1}, \cdots, y_{n}\right)=\left(y_{1}, \cdots, y_{n}, y_{0}\right)$.

THEOREM VII. 2. Let $F$ be a TVS homeomorphic to $X^{\infty}$, where $X=$ $\left(X, x_{0}\right)$ is a pointed locally compact space. Let $B$ be a paracompact space such that $B \times F$ is a $k$-space. Then (a) any locally trivial bundle with base $B$ and fiber $F$ is trivial, and (b) if, also, $F$ has a basis at the origin of $R B S$ sets, then any microbundle with base $B$ and fiber $F$ is trivial.

Proof OF VI. 2. The proof of (a) is like the proof of Theorem 1 in [3], using [8, Corollary II. 5] in place of [3, Lemma 2.3]. The proof of (b) is like the proof [3, Theorem 2] using [8, Corollary II. 5] and our Lemma VII. 1 in place of [3, Lemma 4.1] and [3, Lemma 4.2].

Lemma VII. 3. If $F=B^{*}\left(b^{*}\right)$ or $R^{\infty}$ and if $M$ is a paracompact $F$-manifold, then $M \times F$ is a paracompact $F$-manifold.

Proof of VII. 3. We may assume $M$ is connected. By Theorem II. 1 and Lemma V. 2, $F \cong F \times F$. Thus, $M \times F$ is an $F$-manifold. Since $F$ and $M$ are $\sigma$ compact and regular (Propositions III. 1 and III. 2), so is $M \times F$. Thus, $M \times F$ is regular Lindelöf, and, hence [5, p. 174], paracompact.

Proof of Theorem II. 5. By Theorem II. $1, B^{*}\left(b^{*}\right) \cong Q^{\infty}$. By Theorem IV. 1 both $B^{*}\left(b^{*}\right)$ and $R^{\infty}$ have a basis at the origin consisting of RBS sets. By Lemma VII. 3, $M \times F$ is a paracompact (and connected) $F$-manifold. Thus, by Proposition III. $2 M \times F$ is a CDLCMS, and hence a $k$-space. Theorem II. 5 now follows from Theorem VII. 2.

Proof of Theorem II. 6. By Theorem II. $3 f(M)$ is a neighborhood retract of $N$. The proof of Theorem II. 6 proceeds just as the proof of Theorem 2 in [11]. In place of "the theorem of [10]" used in [11], we appeal to Theorem II. 5. In place of "Lemma 2.1 of [10]" we use the appropriate analogue, proved easily using our Propositions IV. 3 and IV. 4.

Proof of Corollary II. 7. By Theorem II. 1 we may assume $F=R^{\infty}$ or $F=l_{2}(b)$. The result then follows from Theorems II. 2 (b), II. 6 and Lemma V. 2.

VIII. Proof of Theorem II. 8. In this section let $E$ denote either $R^{\infty}$ or $l_{2}(b)$.

LEMMA VIII. 1. Let $M$ and $N$ be paracompact, connected E-manifolds, $g: M \rightarrow N$ a closed embedding. Then $(g, 0): M \rightarrow N \times E$ is a closed, linearly split embedding. 
Proof of VIII. 1. Clearly $(g, 0)$ is a closed embedding. By Theorem II. 2 (b) there is a closed, linearly split embedding $h: M \rightarrow E$. By Propositions III. 2 and III. 3 there is a map $\Lambda: N \rightarrow E$ such that $\Lambda=h g^{-1}$ on $g(M)$. By Theorem II. 3 there is a neighborhood $W$ of $h(M)$ in $E$ and a retraction $r: W \rightarrow h(M)$. Let $\Gamma=g h^{-1} r: W \rightarrow N$. Fix $\left(g\left(m_{0}\right), 0\right) \in(g, 0)(M)$. Since $h$ is linearly split at $m_{0}$, there is an open set $V \subset W$ such that $h\left(m_{0}\right) \in V$ and a homeomorphism $\phi:(V$, $V \cap h(M)) \rightarrow\left(V_{1} \times V_{2}, V_{1} \times 0\right)$, where $V_{i}$ is open in $E_{i}, E_{i}$ a closed linear subspace of $E, i=1,2$, and $E$ is linearly homeomorphic to $E_{1} \times E_{2}$. By Theorem IV. 1 and Proposition IV. 3 there is a neighborhood $U$ of $g\left(m_{0}\right)$ in $N$ and a homeomorphism $j:\left(U, g\left(m_{0}\right)\right) \rightarrow(E, 0)$. Define $\alpha: N \times E \rightarrow E$ by $\alpha(x, y)=$ $\Lambda(x)+y$. Choose neighborhoods $\mathrm{O}_{1}$ of $g\left(m_{0}\right)$ in $N$ and $\mathrm{O}_{2}$ of $0 \in E$ such that $\alpha\left(O_{1} \times O_{2}\right) \subset V$ and then neighborhoods $O_{3}$ of $g\left(m_{0}\right)$ in $O_{1} \cap U$ and $O_{4}$ of 0 in $\mathrm{O}_{2}$ such that $\Gamma \circ \alpha\left(\mathrm{O}_{3} \times \mathrm{O}_{4}\right) \subset U$. Define $\gamma: \mathrm{O}_{3} \times \mathrm{O}_{4} \rightarrow E \times\left(V_{1} \times V_{2}\right)$ by

$$
\gamma(x, y)=(j(x)-j \Gamma(\Lambda(x)+y), \phi(\Lambda(x)+y)) .
$$

Then $\gamma$ is a homeomorphism with inverse

$$
\gamma^{-1}(a, b)=\left(j^{-1}\left[a+j \Gamma \phi^{-1}(b)\right], \phi^{-1}(b)-\Lambda j^{-1}\left[a+j \Gamma \phi^{-1}(b)\right]\right) .
$$

One can show that the expression defining $\gamma^{-1}$ is defined (and continuous) on a neighborhood of each $\gamma(x, y) \in \gamma\left(\mathrm{O}_{3} \times \mathrm{O}_{4}\right)$. Thus $\gamma\left(\mathrm{O}_{3} \times \mathrm{O}_{4}\right)$ is open in $E \times$ $\left(E_{1} \times E_{2}\right)$. Since

$$
\gamma\left[\left(\mathrm{O}_{3} \times \mathrm{O}_{4}\right) \cap(\mathrm{g}, 0)(\mathrm{M})\right]=\gamma\left(\mathrm{O}_{3} \times \mathrm{O}_{4}\right) \cap\left(0 \times E_{1} \times 0\right)
$$

and $E \times E$ is linearly homeomorphic to $E$, we obtain a linear splitting for $(g, 0)$ at $\left(g\left(m_{0}\right), 0\right)$.

Lemma VIII. 2. Let $M, N$ and $E$ be as in Lemma VIII. 1. Let $A \subset M$ be closed, and let $g: M \rightarrow N$ be a map such that $g$ restricted to $A$ is a closed embedding. Let $V$ be any neighborhood of $0 \in E$. Then there is a closed linearly split embedding $\tilde{g}: M \rightarrow N \times E$ such that $\tilde{g}(a)=(g(a), 0)$ for every $a \in A, \tilde{g}(M) \subset$ $g(M) \times V$, and $\tilde{g}$ is homotopic to $g \times 0$ in $g(M) \times V$.

Proof of VIII. 2. By Lemma V. 2 there is a linear homeomorphism $\tau: E \times$ $E \rightarrow E$. Let $O$ be a convex neighborhood of $0 \in E$ such that $\tau(O \times O) \subset V$ (see Theorem IV. 1). Let $W$ be a circled, convex neighborhood of $0 \in E$ such that $W+W \subset 0$. By Theorem II. 2(a) there is a closed embedding $h: M \rightarrow E$ with $h(M) \subset W$. By Propositions III. 2 and III. 3 there is a map $\Lambda: N \rightarrow E$ such that $\Lambda=h g^{-1}$ on $g(A)$ and $\Lambda(N) \subset W$. Define $\bar{g}: M \rightarrow N \times E$ by $\bar{g}(m)=(g(m)$, $\Lambda g(m)-h(m))$. Then $\bar{g}$ is a closed embedding with $\bar{g}(M) \subset g(M) \times 0$. By Lemma VIII. $1,(\bar{g} \times 0): M \rightarrow N \times E \times E$ is a closed, linearly split embedding. 
Then $\tilde{g}=\left(\operatorname{id}_{N} \times \tau\right) \circ(\bar{g} \times 0): M \rightarrow N \times E$ is the desired embedding.

LEMMA VIII. 3. Let $V$ be a convex, radially bounded neighborhood of the origin in TVS $L, x_{0} \in V$. Then there is an invertible isotopy $\rho: L \times I \rightarrow L \times I$ such that $\rho_{0}=\mathrm{id}, \rho_{1}(0)=x_{0}$, and $\rho=\mathrm{id}$ on $(L \backslash V) \times I$.

Proof OF VIII. 3. Assume $x_{0} \neq 0$. Let $R_{x_{0}}$ be the ray in $L$ through $x_{0}$, and let $\epsilon>0$ be such that $R_{x_{0}} \cap V=\left\{r x_{0} \mid 0 \leqslant r<1+\epsilon\right\}$. Let

$$
\begin{array}{ll}
V_{1}=(\epsilon / 8(1+\epsilon)) V, & V_{2}=(\epsilon / 4(1+\epsilon)) V, \\
V_{3}=[(1+\epsilon / 4) /(1+\epsilon)] V, & V_{4}=[(1+\epsilon / 2) /(1+\epsilon)] V .
\end{array}
$$

Each $V_{i}$ is a RBS set, and by Theorem IV. 5 there is an invertible isotopy $H: L \times$ $I \rightarrow L \times I$ such that $H_{0}=$ id, $H_{1}$ is ray preserving, $H_{1}\left(V_{2}\right)=V_{3}$, and $H$ is the identity on $\left(L \backslash V_{4}\right) \times I$. Let $\alpha: L \times I \rightarrow L \times I$ be the homeomorphism $\alpha(x, t)=$ $\left(x+t(\epsilon / 4) x_{0}, t\right)$. Since $(\epsilon / 4) x_{0} \in \operatorname{Bd}\left(V_{2}\right), H(\alpha(0,1))$ must be $(1+\epsilon / 4) x_{0}$, the unique point on $\operatorname{Bd}\left(V_{3}\right) \cap R_{x_{0}}$. The required isotopy is $\rho=\alpha^{-1} \circ H \circ \alpha$.

Proof of Theorem II. 8. Let $M, N, F, f, g: M \rightarrow N \times\{0\} \subset N \times F$ and $V$ be as in Theorem II. 8. By Theorems II. 1 and IV. 1 we may assume $F$ is $R^{\infty}$ or $l_{2}(b)$ and that $V$ is convex and radially bounded. By Lemma VII. $3 N \times F$ is a paracompact, connected $F$-manifold. Let $\tau: F \times F \times F \rightarrow F$ be a linear homeomorphism. Let $W$ be a convex, radially bounded neighborhood of $0 \in F$ such that $\tau(W \times W \times W) \subset V, x_{0} \in W \backslash\{0\}$. Regard $g$ as mapping into $N$, and define $g^{\prime}: M \rightarrow N \times F$ by $g^{\prime}(m)=\left(g(m), x_{0}\right)$. By Lemma VIII. 1 and Theorem II. 3 there is a neighborhood $O$ of $f(M)$ in $N \times W$ and a retraction $r: 0 \rightarrow f(M)$. We may assume 0 is connected. Regard $I \subset F$, and let $r^{\prime}: 0 \times F \rightarrow O \times I$ be a retraction. Since $g^{\prime}$ is homotopic to $f$ in $N \times W$, there is a homotopy $\lambda: 0 \times I \rightarrow$ $N \times W$ with $\lambda_{0}=f f^{-1} r=r$ and $\lambda_{1}=g^{\prime} f^{-1} r$.

Consider $\lambda r^{\prime}: 0 \times F \rightarrow N \times F$. By Lemmas VII. 3 and VIII. 2 there is a closed split embedding $k: 0 \times F \rightarrow N \times F \times F$ with $k(O \times F) \subset N \times W \times W$, $k(f(m), 0)=(f(m), 0)$, and $k(f(m), 1)=\left(g^{\prime}(m), 0\right)$ for every $m \in M$. By Theorem II. 6 there is an open embedding $\tilde{k}: 0 \times F \times F \rightarrow N \times F \times F \times F$ with $\widetilde{k}(x, y, 0)$ $=(k(x, y), 0)$, all $(x, y) \in 0 \times F$. By normality we can find an open set $U$ in $N \times F \times F$ with

$$
\tilde{k}(0 \times F \times\{0\}) \subset U \subset \bar{U} \subset \tilde{k}(0 \times F \times F) \cap(N \times W \times W \times W),
$$

$\bar{U}$ the closure of $U$ in $N \times F \times F \times F$. By Propositions IV. 3 and IV. 4 there is an open embedding $h: 0 \times F \times F \rightarrow \tilde{k}^{-1}(U)$ such that $h$ is the identity on $0 \times F \times\{0\}$ and $h(0 \times F \times \bar{W})$ is closed in $0 \times F \times F$. This implies $\widetilde{k} h(O \times F \times \bar{W})$ is closed in $\bar{U}$.

Define a homeomorphism $j: 0 \times F \times F \times I \rightarrow 0 \times F \times F \times I$ as follows: 
Let $\gamma=\gamma_{W}: F \backslash\{0\} \rightarrow[0, \infty)$ be as in Lemma IV. 2. Let $\delta:[0, \infty) \rightarrow I$ be a map such that $\delta([0,1 / 2])=0$ and $\delta([1, \infty))=1$. Then $\delta^{\prime}: F \rightarrow I$ defined by $\delta^{\prime}=\delta \gamma$ on $F \backslash\{0\}$ and $\delta^{\prime}(0)=0$ is continuous. Note that $\delta^{\prime}(F \backslash W)=1$. Let $j(x, y, z, t)=\left(x, y+t\left(1-\delta^{\prime}(z)\right), z, t\right)$. Define a homeomorphism $\hat{j}: N \times F \times$ $F \times I \rightarrow N \times F \times F \times F \times I$ by

$$
\hat{j}=\left\{\begin{array}{l}
(\tilde{k} h \times \mathrm{id}) \circ j \circ(\widetilde{k} h \times \mathrm{id})^{-1}, \quad \text { on } \tilde{k} h(0 \times F \times F) \times I, \\
\mathrm{id}, \quad \text { off }(\tilde{k} h \times \mathrm{id})(0 \times F \times \bar{W} \times I) .
\end{array}\right.
$$

Note that $(\hat{j})_{0}=\mathrm{id}, \hat{j}(f(m), 0,0,1)=\left(g^{\prime}(m), 0,0,1\right)$, and $\hat{j}$ is the identity off $N \times W \times W \times W \times I$. Let

$$
\bar{\tau}=\text { id } \times \tau \times \text { id: } N \times F \times F \times F \times I \rightarrow N \times F \times I .
$$

Then $j^{\prime}=\bar{\tau} \circ \hat{j} \circ \bar{\tau}^{-1}: N \times F \times I \rightarrow N \times F \times I$ is an invertible isotopy with $\left(j^{\prime}\right)_{0}=\mathrm{id},\left(j^{\prime}\right)(f(m), 1)=\left(g(m), \tau\left(x_{0}, 0,0\right), 1\right)$, and $j^{\prime}$ the identity on $N \times(F \backslash V)$ $\times I$. An application of Lemma VIII. 3 now gives the required invertible isotopy.

IX. Proof of Theorem II. 9. Let $f: M \rightarrow N, g: N \rightarrow M$ be homotopy inverses. By Theorem II. 1 we may assume that $F=R^{\infty}$ or $F=l_{2}(b)$. By Lemma VIII. 2 there is a closed split embedding $\bar{f}: M \rightarrow N \times F$. Let $\tau: F \times F \rightarrow F$ be a linear homomorphism (see Lemma V. 2), and define $\hat{f}: M \times F \rightarrow N \times F$ by $\hat{f}(m, x)=\left(\bar{f}_{1}(m), \tau\left(\bar{f}_{2}(m), x\right)\right)$, where $\bar{f}=\left(\bar{f}_{1}, \bar{f}_{2}\right)$. Then $\hat{f}$ is a closed split embedding homotopic to $f \times$ id. Similarly, define $\hat{g}: N \times F \rightarrow M \times F$.

The proof now proceeds as the proof of Theorem 4, in [11]. Use our Theorem II. 6 where Henderson applies his Theorem 2. In place of his $F_{1}$, substitute a fixed RBS set $A \subset F$. In place of his "Lemma 1.2 of [10]" use our Propositions IV. 3 and IV.4. Substitute an arbitrary RBS $V$ for his " $F_{\alpha}$ ". Finally, apply our Theorem II. 8 and Lemma IV. 6 where he applies his Theorem 3 and Lemma 5.1, respectively.

\section{Proof of Theorem II. 10.}

LEMmA X. 1. Let $F=R^{\infty}$ or $B^{*}\left(b^{*}\right)$. Then any open subset of $F$ has the homotopy type of a $\mathrm{CW}$-complex.

Proof of X. 1. Let $W \subset F$ be open. Write $F=\bigcup\left\{C_{i} \mid i=1,2, \cdots\right\}$, where each $C_{i}$ is a compact, convex, metric space, $C_{i} \subset C_{i+1}$, and $F=\lim _{\rightarrow}\left\{C_{i}\right\}$. (For $F=B^{*}\left(b^{*}\right)$ let $C_{i}=\left\{x \in B^{*} \mid\|x\| \leqslant i\right\}$. Then $C_{i}$ is compact and $\overrightarrow{m e t r i c}$ by Alaoglu's theorem [6, pp. 424, 426]. For $F=R^{\infty}$, let $C_{i}=\left\{x \in R^{i} \mid\|x\| \leqslant i\right\}$.) Then $W=\lim _{\rightarrow}\left\{W \cap C_{i}, j_{i}\right\}$, where $j_{i}: W \cap C_{i} \rightarrow W \cap C_{i+1}$ is the inclusion. By Dugundji's extension theorem, [5, p. 188], each $C_{i}$ is an AR (metric). Hence, each $W \cap C_{i}$ is an ANR (combine Proposition 6.5 and Proposition 7.9 in Chapter 
III of [12]). By Corollary 6.4 of [7], $W$ has the homotopy type of an ANR and, hence [7, Lemma 6.6], of a CW-complex.

Theorem II. 10 will now follow from the following theorem.

THEOREM X 2. Let $M$ be a paracompact manifold modelled on the TVS $L$, where each open subset of $L$ has the homotopy type of a CW-complex. Then $M$ has the homotopy type of a CW-complex.

The proof of X. 2 uses the following definition and theorem of T. tom Dieck [4] :

DEFINITION [4]. A covering $\left\{E_{\alpha} \mid \alpha \in A\right\}$ of a space $E$ is called numerable if there is a locally finite partition of unity $\left\{t_{\alpha} \mid \alpha \in A\right\}$ such that

$$
\overline{t_{\alpha}^{-1}((0,1])} \subset E_{\alpha} \text {, each } \alpha .
$$

Theorem [4, ThEorem 4]. Let $U=\left\{X_{\alpha} \mid \alpha \in A\right\}$ be a numerable covering of a space $X$. If, for each nonempty $\sigma \subset A, \bigcap\left\{X_{\alpha} \mid \alpha \in \sigma\right\}$ has the homotopy type of a CW-complex, then $X$ has the homotopy type of a CW-complex.

Proof of Theorem X 2. Let $\left\{O_{\alpha} \mid \alpha \in A\right\}$ be a locally finite cover of $M$ by sets homeomorphic to open subsets of $L$. Since $M$ is paracompact, $\left\{O_{\alpha}\right\}$ is numerable. Let $\sigma \subset A$ be such that $\bigcap\left\{\mathrm{O}_{\alpha} \mid \alpha \in \sigma\right\} \neq \varnothing$. Then $\sigma$ must be finite, so $\bigcap\left\{0_{\alpha} \mid \alpha \in \sigma\right\}$ is homeomorphic to an open subset of $F$, and, hence, has the homotopy type of a CW-complex. Theorem X. 2 now follows from the theorem of T. tom Dieck.

XI. Proof of Theorem II. 11. If $U$ is an open subset of $Q^{\infty}$ we write $U^{n}$ for $U \cap Q^{n}$.

LEMMA XI. 1. Let $U$ be an open subset of $Q^{\infty}$. Then $U$ has a basis of open subsets $\left\{W_{\alpha} \mid \alpha \in \mathscr{Q}\right\}$ such that each $W_{\alpha}$ is convex and each $U_{\alpha} \cap Q^{n}$ is relatively compact in $U^{n}$.

Proof OF XI. 2. Let

$$
C=\left\{W_{\alpha}=\left(W_{\alpha_{1}} \times W_{\alpha_{2}} \times \cdots\right) \cap Q^{\infty} \mid W_{\alpha_{i}} \subset Q\right. \text {, }
$$

$W_{\alpha_{i}}$ convex and open, $\overline{W_{\alpha}} \subset U$ \}

Here $\bar{W}_{\alpha}$ is the closure of $W_{\alpha}$ in $Q^{\infty}$. By [8, Proposition II. 1], and since $Q^{\infty}$ is regular, it follows that $C$ is a basis for $U$. The closure of each $W_{\alpha} \cap Q^{n}$ in $U^{n}$ equals the closure of $W_{\alpha} \cap Q^{n}$ in $Q^{n}$ and is hence compact.

Definitions. A map $f: X \rightarrow Y$ is proper if the inverse image (under $f$ ) of any compact subset of $Y$ is compact. Let $f$ and $g$ map $X$ into $Y$. Let $U$ be any open cover of $Y$. Then $f$ and $g$ are $U$-close if for each $x \in X$ there is a $U \in U$ with $\{f(x), g(x)\} \subset U$. If $H: X \times I \rightarrow Y$ is a homotopy, then $H$ is limited by 
$U$ if, for each $x \in X, H(\{x\} \times I) \subset U$ for some $U \in U$.

Lemma XI. 2 (cF. [1, Lemma 2.2]). Let $X$ and $Y$ be spaces, and let $U$ be any locally finite cover of $Y$ by relatively compact open sets. Let $f$ and $g$ be maps of $X$ into $Y$ such that $f$ and $g$ are U-close and $f$ is proper. Then $g$ is also proper.

Proof of XI. 2. Let $U=\left\{U_{\alpha}\right\}$. Let $A \subset Y$ be compact. Since $\left\{U_{\alpha}\right\}$ is locally finite, $A$ meets only finitely many of the $U_{\alpha}$ 's, say $U_{1}, \cdots, U_{n}$. Let $C=\bigcup\left\{\bar{U}_{i} \mid i=1, \cdots, n\right\}$. Let $x \in g^{-1}(A)$. There is an $\alpha$ with $\{g(x), f(x)\} \subset$ $U_{\alpha}$. But then $U_{\alpha}$ must be $U_{i}$, some $i \leqslant n$. Thus, $f\left(g^{-1}(A)\right) \subset C$, which is compact. Hence, $g^{-1}(A)$ is a closed subset of the compact $f^{-1}(C)$ and is therefore compact.

The following theorem is implicit from the Addendum of [2].

THEOREM (ANDERSON-SCHORI [2]). Let $O \subset Q$ be an open set, $U$ any open cover of 0 . Then there is a homeomorphism $h: Q \times 0 \rightarrow 0$ such that for every $(x, y) \in Q \times 0$ there is a $U \in U$ with $\{y, h(x, y)\} \subset U$.

Proof of Theorem II. 11. By Theorem II. 1 we need only show that $U \times Q^{\infty} \cong U$, where $U$ is open in $Q^{\infty}$. Let $i_{n}: U^{n} \rightarrow U^{n+1}$ and $j_{n}: U^{n} \times Q^{n} \rightarrow$ $U^{n+1} \times Q^{n+1}$ be the natural inclusions. We first construct a sequence of homeomorphisms $h_{n}: U^{n} \rightarrow U^{n} \times Q^{n}, n \geqslant 1$, such that $h_{n+1} i_{n}$ and $j_{n} h_{n}$ are properly homotopic. By Proposition III. $1 U$ is paracompact. Thus, by Lemma XI. 1, there is a locally finite cover $V$ of $U$ such that $V^{n}$ is relatively compact in $U^{n}$ for each $V \in V$ and then a refinement $C$ of $V$ consisting of convex open sets. By [5, Theorem 3.5, p. 168] there is an open barycentric refinement $W$ of $C$. Let $W^{n}=$ $\left\{W \cap Q^{n} \mid W \in W\right\}$. Let $p_{n}: U^{n} \times Q^{n} \rightarrow U^{n}$ be the projection. By the theorem of Anderson and Schori there is a homeomorphism $h_{n}: U^{n} \rightarrow U^{n} \times Q^{n}$ such that, for each $x \in U^{n},\left\{x, p_{n} h_{n}(x)\right\}$ is contained in some element of $W^{n}$. Consider the diagram

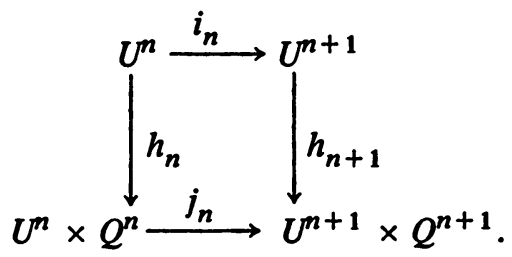

Let $x \in U^{n}$. There is a $W_{1} \in W$ such that $\left\{x, p_{n} h_{n}(x)\right\} \subset W_{1}^{n}$, and hence $\left\{i_{n}(x)\right.$, $\left.p_{n+1} j_{n} h_{n}(x)\right\} \subset W_{1}^{n+1}$. There is a $W_{2} \in W$ such that $\left\{i_{n}(x), p_{n+1} h_{n+1} i_{n}(x)\right\} \subset$ $W_{2}^{n+1}$. Thus, $\left\{p_{n+1} h_{n+1} i_{n}(x), p_{n+1} j_{n} h_{n}(x)\right\}$ is contained in the star of $i_{n}(x)$ with respect to $W$, and hence in some $C \in C$. Since $C$ is convex, we can define a homotopy $H: U^{n} \times I \rightarrow U^{n+1} \times Q^{n+1}$ by 


$$
H(x, t)=(1-t) j_{n} h_{n}(x)+t h_{n+1} i_{n}(x) .
$$

Then $H_{0}=j_{n} h_{n}$ which is proper, and $H_{1}=h_{n+1} i_{n}$. Also, $H$ is limited by $\left\{V^{n+1} \times Q^{n+1} \mid V \in V\right\}$, and it follows, using Lemma XI. 2, that $H$ is proper.

We now define a sequence of homeomorphisms $g_{n}: U^{n} \rightarrow U^{n} \times Q^{n}$ such that $g_{n+1} i_{n}=j_{n} g_{n}$. Let $g_{1}=h_{1}$. Then $j_{1} g_{1}$ and $h_{2} i_{1}$ are properly homotopic maps onto $Z$-sets in $U^{2} \times Q^{2}$. By the main theorem of [1] there is an invertible isotopy $G$ : $U^{2} \times Q^{2} \times I \rightarrow U^{2} \times Q^{2} \times I$ such that $G_{0}=$ id and $G_{1} h_{2} i_{1}=j_{1} g_{1}$. Let $g_{2}=G_{1} h_{2}$. Then $g_{2} i_{1}=j_{1} g_{1}$, and $j_{2} g_{2}$ is properly homotopic to $h_{3} i_{2}$. Continuing inductively we obtain the desired sequence $\left\{g_{n} \mid n=1,2, \cdots\right\}$. The sequence $\left\{g_{n}\right\}$ induces a homeomorphism

$$
g: \lim _{\rightarrow}\left\{U^{n}, i_{n}\right\} \rightarrow \lim _{\rightarrow}\left\{U^{n} \times Q^{n}, j_{n}\right\} .
$$

But $\lim _{\rightarrow}\left\{U^{n}, i_{n}\right\}=U$, and $\lim _{\rightarrow}\left\{U^{n} \times Q^{n}, j_{n}\right\}=U \times Q^{\infty}$. (The last assertion is a consequence of $[8$, Corollary III. 1].)

\section{REFERENCES}

1. R. D. Anderson and T. A. Chapman, Extending homeomorphisms to Hilbert cube manifolds, Pacific J. Math. 38 (1971), 281-293. MR 47 \#7749.

2. R. D. Anderson and R. Schori, Factors of infinite-dimensional manifolds, Trans. Amer. Math. Soc. 142 (1969), 315-330. MR 39 \#7631.

3. T. A. Chapman, Locally-trivial bundles and micro-bundles with infinite-dimensional fibers, Proc. Amer. Math. Soc. 37 (1973), 559-602. MR 47 \#1081.

4. Tammo tom Dieck, Partitions of unity in homotopy theory, Compositio Math. 23 (1971), 159-167. MR 45 \#2702.

5. J. Dugundji, Topology, Allyn and Bacon, Boston, Mass., 1966. MR 33 \#1824.

6. N. Dunford and J. T. Schwartz, Linear operators. I: General theory, Pure and Appl. Math., vol. 7, Interscience, New York, 1958; 2nd printing, Interscience, New York, 1964. MR 22 \#8302.

7. V. L. Hansen, Some theorems on direct limits of expanding sequences of manifolds, Math. Scand. (1971), 5-36. MR 47 \#7751.

8. R. E. Heisey, Contracting spaces of maps on the countable direct limit of a space, Trans. Amer. Math. Soc. 193 (1974), 389-412.

9. - Partitions of unity and a closed embedding theorem for $\left(C^{p}, b^{*}\right)$-manifolds, Trans. Amer. Math. Soc. 206 (1975), 281-294.

10. D. W. Henderson, Micro-bundles with infinite-dimensional fibers are trivial, Invent. Math. 11 (1970), 293-303. MR 43 \#8092.

11. Stable classification of infinite-dimensional manifolds by homotopy-type, Invent. Math. 12 (1971), 48-56. MR 44 \#7594.

12. Sze-Tsen Hu, Theory of retracts, Wayne State Univ. Press, Detroit, 1965. MR 31 \#6202.

13. J. L. Kelley and I. Namioka, Linear topological spaces, University Ser. in Higher Math., Van Nostrand, Princeton, N. J., 1963. MR 29 \#3851.

14. R. S. Palais, Banach manifolds of fiber bundle sections, Actes Congr. Internat. Math., Nice, 1970, vol. 2, Gauthier-Villars, Paris, 1971, pp. 243-249.

DEPARTMENT OF MATHEMATICS, CORNELL UNIVERSITY, ITHACA, NEW YORK 14850

Current address: Department of Mathematics, Vanderbilt University, Nashville, Tennessee 37235 